\title{
Editorial
}

\author{
JULIAN E. SALE ${ }^{1}$, TIMOTHY M. COX ${ }^{2}$ \\ ${ }^{1}$ MRC Laboratory of Molecular Biology, UK, and ${ }^{2}$ Department of Medicine, University of Cambridge, UK
}

\section{ERM Discovery}

Expert Reviews in Molecular Medicine has a welldeserved reputation for authoritative reviews on an eclectic range of topics in contemporary molecular medicine and on the translation of discoveries in basic molecular pathophysiology to clinical practice.

Along with a number of other eminent Cambridge review journals, including the Quarterly Review of Biophysics, Expert Reviews in Molecular Medicine has recently expanded its remit to include the publication of primary research papers in a 'Discovery' section of the Journal. We hope that this section will become an important feature of the Journal by providing a channel for the communication of cutting edge new work in the diverse areas covered by the Journal. The scope mirrors that of the 'Reviews' section of Journal, focussing on studies providing mechanistic insights or diagnostic advances.

We have already received strong submissions on a range of topics and the first papers have now been published online. The first two accepted manuscripts examined aspects of the diagnosis of Fragile X syndrome. Rajan-Babu and colleagues presented a simplified and cost-effective test for measuring the triplet repeat expansion in the FMR1 gene, mutated in Fragile X Syndrome (Rajan-Babu et al 2015, ERMM 17, e7), while Godler and colleagues demonstrated a novel approach to determine skewed X-chromosome inactivation both in females with full fragile $\mathrm{X}$ syndrome mutation and in sex chromosome aneuploidies (Godler et al. 2015, ERMM 17, e13). Two further papers due to appear soon examine the role of microRNA 124 in suppressing seizure activity and the mechanism by which Calpain 3 deficiency affects skeletal muscle function. These papers all align extremely well with our hopes for the Discovery section by providing molecular insights to the mechanisms of disease and to its diagnosis.

We would like to encourage more submissions in the same vein. To facilitate this, we are happy to receive manuscripts for review in any format that allows an initial assessment of the work, including the submission of a single PDF containing both text and figures, which we hope will save time for authors. We do, however, encourage authors to provide a concise cover letter explaining the principle findings of the work and its importance within its subject area. We aim to review papers thoroughly but rapidly. The editor will determine within a week whether the paper should be sent for in-depth review. Following peer review we will provide clear editorial guidance on the modifications or additional work that we believe will be necessary to bring the paper to publication. Once accepted, the CUP production team will ensure that the online presentation of articles maximises the impact of the data on a wide range of electronic platforms and all papers are published under a Gold open access model to ensure the widest possible readership.

We look forward to receiving submissions of your most interesting work, and to the continuing growth and development of the Journal. 